\title{
Osteoarthritis and Management
}

\section{Basem Al-Omari*}

School of Health and Life Science, University of Northumbria, UK
ISSN: 2576-8875

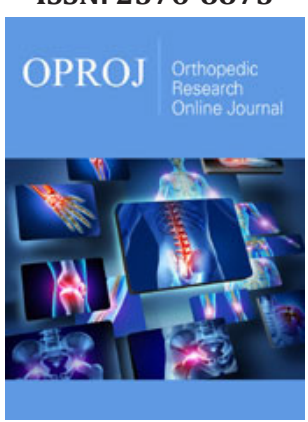

${ }^{* 1}$ Corresponding author: Basem Al-Omari, Faculty of Health and Life Sciences, Coach Lane Campus East, University of Northumbria, UK

Submission: 憏June 21, 2019

Published: 䟧June 27, 2019

Volume 5 - Issue 5

How to cite this article: Basem $\mathrm{Al}$-Omari. Osteoarthritis and management. Ortho Res Online J. 5(5). OPROJ.000622.2019. DOI: 10.31031/OPROJ.2019.05.000622

Copyright@: Basem Al-Omari, This article is distributed under the terms of the Creative Commons Attribution 4.0 International License, which permits unrestricted use and redistribution provided that the original author and source are credited.

\section{Abstract}

Osteoarthritis $(\mathrm{OA})$ is a degenerative disease that causes pain, limitation of movement, and disability. The diagnosis of OA requires clinical examination, pathology, and/or radiography. OA has no cure, the current available treatment focuses on pain management and improving the quality of life. This treatment includes non-pharmacological treatment such as exercises and physiotherapy, pharmacological such as acetaminophen, Nonsteroidal Anti-Inflammatory Drugs (NSAIDs) and opioids, and surgical interventions such as joint replacement.

Keywords: Osteoarthritis; Arthritis; Joint pain; Degenerative disease

\section{Osteoarthritis Background}

$\mathrm{OA}$ is a chronic disabling and intermittently or slowly progressive joint degenerative disease [1]. It is the most common form of arthritis; it involves degradation of the articular cartilage, synovium, and subchondral bones [2] causing joint pain, functional limitation and reduced quality of life [3]. OA can affect any joint. However, the knee, hip, hand and spine are the most common sites of $\mathrm{OA}$, but it can also occur in the ankles and wrists [4].

OA can be diagnosed clinically, pathologically, or radiographically which is the most frequent definition for OA [5]. Initially, clinicians may define OA by examining the patients, signs and symptoms, and risk factors. The most common radiographic grading system for $\mathrm{OA}$ is Kellgren and Lawrence grade, which score the severity of OA on a scale of 0 to 4 [6]. A score of 2 or above in Kellgren and Lawrence indicates definite radiographic OA [7].

The prevalence of $\mathrm{OA}$ increases with age and it affects as many as $80 \%$ of the population over 75 years of age [8]. Generally, women are at greater risk of OA and have more severe symptoms than men, especially after menopausal age [9]. The World Health Organisation (WHO) estimates that OA affects $9.6 \%$ of men and $18 \%$ of women over 60 years of age [10]. In the United Kingdom (UK), OA is considered to be one of the leading causes of pain and disability [11]. It is also responsible for a large proportion of physician time, hospitalisations, and time lost from work in the Western countries [12]. As life expectancy and the numbers of older people in the population are increasing, it is expected that OA will be the fourth leading cause of disability by the year 2020 [13].

\section{Osteoarthritis Management}

At the present time, there is still no cure for OA [14]. The treatments that are currently available for $\mathrm{OA}$ are aiming for pain reduction, correcting deformities, and improving joint function to enhance the quality of the patient's life $[15,16]$.

Guidelines for the management of OA pain are available from a number of sources e.g. NICE OA guidelines [15] and Zhang et al. [17] recommendations [15,16]. Generally, OA treatment includes non-pharmacological, pharmacological, and surgical interventions [18]. Although non-pharmacological treatments of $\mathrm{OA}$ such as exercise and physiotherapy revealed a modest effect in reducing symptoms, one of their main advantages is that they pose no inherent risks [18]. Therefore, the non-pharmacological treatments are recommended at all levels of OA severity $[15,18]$.

In the UK, the National Institute of Health and Clinical Excellence (NICE) [15] recommend exercise as the core treatment for people with $\mathrm{OA}$, irrespective of age, comorbidity, pain 
severity or disability. This core treatment is a treatment that should be considered for every person with OA. However, as a treatment for OA, exercise is not necessarily or always successful alone [20]. In this case, pharmaceutical treatments for OA are considered.

Pharmaceutical treatments for OA consist of different types of medication including pain relieving and anti-inflammatory medications [20]. Most guidelines recommend acetaminophen (paracetamol) as the initial oral drug [21]. The NICE OA guideline suggests that paracetamol should be offered for pain relief in addition to exercise [15]. In patients who do not experience adequate symptomatic relief with exercise and paracetamol, alternative or additional pharmacological medication such as nonsteroidal antiinflammatory drugs (NSAIDs) should be considered [15,22,23]. NSAIDs are made in different forms and have different methods of administration e.g. oral, topical and rectal. Oral NSAIDs have shown risk of adverse effects in relation to systems such as gastrointestinal, liver and cardio-vasculara, which is higher than paracetamol or topical NSAIDs $[2,20,23]$. Thus, the main UK arbiter of approved effective clinical treatments for use in the National Health Service recommends that topical NSAIDs should be considered ahead of oral NSAIDs, Cyclo-oxygenase 2 (COX-2) inhibitors or opioids $[15,24]$. Although NSAIDs and COX-2 inhibitors may be regarded as a single drug class of 'NSAIDs', NICE OA guidelines continues to use the two terms for clarity, and because of the differences in the side effect profiles. When the combination of paracetamol with NSAIDs fails, then the addition of opioid analgesics is considered $[8,15]$.

Surgical treatment should be the last resource of treatment for OA [15]. Nonetheless, when non-surgical treatments fail and there is prolonged and established functional limitation and severe pain that have a substantial impact on the quality of life, referral for joint replacement surgery is recommended [2].

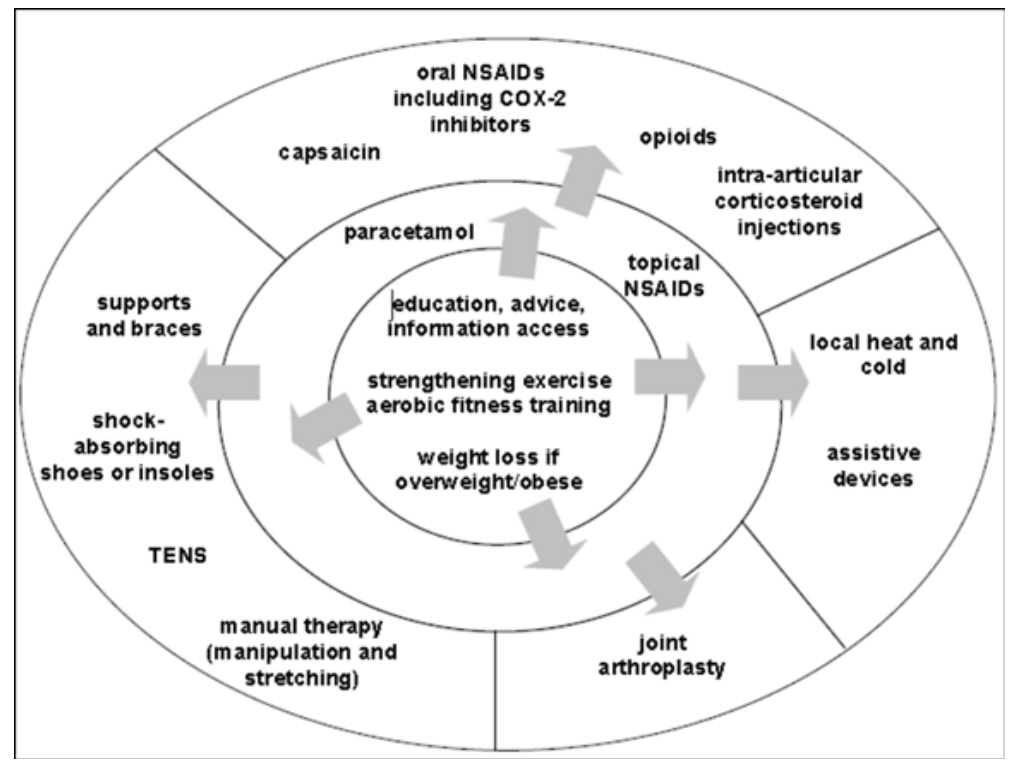

Figure 1: Summary of the treatments adopted from NICE OA guideline [15].

Figure 1 shows the order of treatment to be considered for people with OA as recommended by NICE OA guidelines [15]. The treatments are arranged in the order in which they should be considered for people with OA starting from the central circle. The central circle includes non-pharmaceutical treatment that should be considered for every OA patient at all stages of severity. Going outward from the central circle, the middle circle shows the next treatment that should be considered for OA patients combined with the central circle treatment. Finally, the external circle in the figure includes treatments that should be considered if the treatment in the central and middle circle failed. It is vital that individual needs, risk factors and preferences are considered at every stage of treatment.

\section{Conclusion}

$\mathrm{OA}$ is a chronic disabling disease that causes pain and limitation of movement. It is considered to be one of the leading causes of disability and time lost from work. There is still no cure for OA.
Therefore, symptomatic treatment is essential to reduce pain and improve the quality of life. The first and core treatment for $\mathrm{OA}$ should start with non-pharmacological non-surgical management such as exercises and physiotherapy. If exercise and physiotherapy are not successful or patients are still experiencing pain or movement limitation, further pharmacological treatment could be used in conjunction with the core treatment. Surgical treatment should always be the last resource for OA treatment.

\section{Future directions}

Pharmacological treatment for OA, such as NSAIDs and opioids have several risks of adverse effects that may have an impact on patients' health. Therefore, it is vital that patients are informed of all adverse effects associated with these medications. This will aid the shared decision-making process between patients and their clinicians and will facilitate an informed decision made by the patients to opt for the pharmacological treatment or not. 


\section{References}

1. Yang KGA, Saris DBF, Dhert WJA, Verbout AJ (2004) Osteoarthritis of the knee: current treatment options and future directions. Current Orthopaedics 18: 311-320.

2. Rivero-Santana A, Perestelo-Perez L, Perez-Ramos J, Gonzalez-Lorenzo M, Serrano-Aguilar P (2012) Treatment preferences in patients with knee or hip osteoarthritis: An overview. In Osteoarthritis - Diagnosis, Treatment and Surgery, Shanghai, InTech China, China, pp. 227-248.

3. Cooper C, Adachi JD, Bardin T, Berenbaum F, Flamion B (2013) How to define responders in osteoarthritis. Current Medical Research and Opinion 29(6): 719-729.

4. Arden N, Nevitt MC (2006) Osteoarthritis: epidemiology. Best Practice \& Research Clinical Rheumatology 20(1): 3-25.

5. Zhang Y, Jordan JM (2010) Epidemiology of osteoarthritis. Clin Geriatr Med 26(3): 355-369.

6. Neogi T, Zhang Y (2011) Osteoarthritis prevention. Current Opinion in Rheumatology 23: 185-191.

7. Kellgren JH, Jeffrey MR, Ball J (1963) The epidemiology of chronic rheumatism: Atlas of standard radiographs, Blackwell Scientific, Oxford, England.

8. Bijlsma J (2002) Analgesia and the patient with osteoarthritis. American Journal of Therapeutics 9(3): 189-197.

9. Srikanth VK, Fryer JL, Zhai G, Winzenberg TM, Hosmer D, et al. (2005) A meta-analysis of sex differences prevalence, incidence and severity of osteoarthritis. Osteoarthritis and cartilage / OARS, Osteoarthritis Research Society 13(9): 769-781.

10. Wittenauer R, Smith L, Aden K (2013) Update on 2004 Background Paper, BP 6.12 Osteoarthritis.

11. Conaghan PG, Dickson J, Grant RL, Guideline Development Group (2008) Care and management of osteoarthritis in adults: summary of NICE guidance. BMJ 336(7642): 502-503.

12. Bitton R (2009) The economic burden of osteoarthritis. The American Journal of Managed Care 15(8): 230-235.

13. Woolf AD, Pfleger B (2003) Burden of major musculoskeletal conditions. Bulletin of the World Health Organization 81(9): 646-656.
14. Li A, Zhang Y, Lao L, Xin J, Ren K, et al. (2011) Serotonin receptor 2A/C is involved in electroacupuncture inhibition of pain in an osteoarthritis rat model. Evidence-based Complementary and Alternative Medicine 2011: 619650.

15. NICE (2014) Osteoarthritis: care and management. NICE guidline [CG177].

16. Al-Omari B, Frisher M, Croft P, Sim J (2017) Generating individual patient preferences for the treatment of osteoarthritis using Adaptive Choice-Based Conjoint (ACBC) analysis. Rheumatology and Therapy 4(1): 167-182.

17. Zhang W, Nuki G, Moskowitz RW, Abramson S, Altman RD (2010) OARSI recommendations for the management of hip and knee osteoarthritis: pasrt III: Changes in evidence following systematic cumulative update of research published through January 2009. Osteoarthritis and Cartilage / OARS, Osteoarthritis Research Society 18(4): 476-499.

18. Al-Omari B (2017) Patient preferences for the pharmacological treatment of osteoarthritis using adaptive choice-based conjoint (ACBC) analysis: A pilot study. European Journal for Person Centered Healthcare 5(2): 220-224.

19. Scott D, Kowalczyk A (2007) Osteoarthritis of the knee. BMJ Clinical Evidence 1121: 19450299.

20. https://www.nhs.uk/conditions/osteoarthritis/treatment/

21. Al-Omari B, Sim J, Croft P, Frisher M (2015) Patient preferences for the pharmacological treatment of osteoarthritis: a feasibility study using Adaptive Choice-Based Conjoint Analysis (ACBCA). European Journal for Person Centered Healthcare 3(2): 253-260.

22. Bacon TH, Hole JG, North M, Burnett I (2002) Analgesic efficacy of sustained release paracetamol in patients with osteoarthritis of the knee. British Journal of Clinical Pharmacology 53(6): 629-636.

23. Jawad AS (2005) Analgesics and osteoarthritis: are treatment guidelines reflected in clinical practice? American Journal of Therapeutics 12(1): 98-103.

24. Al-Omari B, Frisher M, Croft P, Sim J (2013) Using Adaptive Choice Based Conjoint (ACBC) Analysis to study patients' preferences. Annals of the Rheumatic Diseases 72(3): 567. 